\title{
A discrete-time ATM traffic model with long range dependence characteristics
}

\author{
Tim Daniëls and Chris Blondia \\ Performance Analysis of Telecommunication Systems Research Group \\ Department of Mathematics and Computer Science \\ University of Antwerp \\ Universiteitsplein, 1, B-2610 Antwerp - Belgium \\ e-mail: \{tdaniels, blondia\} @uia.ac.be
}

\begin{abstract}
This paper presents a novel discrete-time ATM traffic model which exhibits a long range dependence character. The process results from the superposition of an infinite number of on/off sources which have an increasing mean on and off period duration. The condition under which the process has the long range dependence property is a simple function of the parameters of the on/off sources. Moreover, the Hurst parameter of the process is given explicitly. By considering the traffic model in a matrix-analytic setting, the behaviour of a queueing system with this traffic as input, can be analyzed. Also the correlation structure is characterized by means of the Index of Dispersion for Counts and its limit is investigated. Numerical examples illustrate the properties of this process.
\end{abstract}

\section{Keywords}

ATM, long range dependence, queueing, D-BMAP

\section{INTRODUCTION}

Recent measurements of LAN traffic (see (Leland, Taqqu, Willinger \& Wilson 1994), (Fowler \& Leland 1991)) and variable bit rate (VBR) video traffic (see (Beran, Sherman, Taqqu \& Willinger 1995)), show their bursty character accross a high range of time scales. Statistical analysis reveals slowly decaying variances and long range dependence of the number of packets arriving in a time slot. This suggest a self-similar behaviour of this type of traffic.

In a discrete-time setting, the long range dependence of a process $X_{t}$ can be characterized in different ways. Let $A_{n}=\sum_{k=1}^{n} X_{k}$ denote the cummulative process and $f(\lambda)$ the spectral density of $X_{n}$. Then $X_{n}$ is said to be long range dependent if one of the following conditions is satisfied (see (Roberts, Mocci \& Virtamo 1996, page 317)) :

$$
\begin{aligned}
& -\operatorname{cov}\left(X_{0}, X_{n}\right) \underset{n^{\beta_{1}}}{\sim}, \beta_{1}=2 H-2 \\
& -\operatorname{var}\left(A_{n}\right) \sim{ }^{\beta_{2}}, \quad \beta_{2}=2 H
\end{aligned}
$$


- $f(\lambda) \sim \lambda^{\beta_{3}}, \beta_{3}=1-2 H$,

where the asymptotics hold for $n \longrightarrow \infty$ and $\lambda \longrightarrow 0$. In these conditions $H$ denotes the Hurst parameter (or self-similarity parameter), with $\left.H \in] \frac{1}{2}, 1\right]$. This Hurst parameter is an essential characteristic of long range dependence traffic.

Conventional traffic models, such as the pure Poisson process, the Markov modulated Poisson-like processes (e.g. MAP, D-MAP, BMAP, D-BMAP, see (Neuts 1989), (Lucantoni 1991), (Blondia 1993)), or fluid flow models (Anick, Mitra \& Sondhi 1982), do not capture this long range dependence and consequently are not appropriate to model this type of traffic. From the measurements it also follows that established views, such as the aggregate traffic becomes smoother as the number of (bursty) sources increases, do not hold any longer. On the contrary, the burstiness of the aggregate traffic increases. These observations have started an intensive research on models which capture the long range dependence nature of the traffic. Examples of such models are the Fractional Brownian Motion, FARIMA process ((Roberts et al. 1996), chaotic maps, etc ...

Unfortunately, queueing analysis results of systems where these traffic models are used are hardly available. An alternative approach has been proposed in (Andersen 1995), (Andersen, Jensen \& Nielsen 1995) and (Robert \& Le Boudec 1995), where Markovian arrival processes (MAP) are used to model self-similarity over several time scales. A fitting algorithm to chose the parameters of the MAP is proposed in (Andersen \& Nielsen n.d.). Another approach to characterize long range dependent traffic is through an infinite superposition of on/off sources which have an on period with a heavy tail. An example can be found in (Likhanov, Tsybakov \& Georganas 1995), where the resulting queue is an M/G/1 system with Pareto service time distribution.

In this paper, we propose a new model for ATM traffic resulting from the superposition of a sequence of on/off sources, with increasing mean on and off periods as the index of the sequence increases. The model has the following characteristics :

(i) It is defined by means of three parameters $(a, b, p)$;

(ii) Under the condition that $a \geq b^{2}$, the resulting process exhibits a long range dependence character;

(iii) The Hurst parameter is given explicitly by $H=\frac{1}{2}\left(\frac{\log b}{\log a-\log b}\right)$;

(iv) The analysis of the queueing system whose input consists of this process can be seen as the limit of a sequence of Markovian queues, which are analytically tractable; (v) The Index of Dispersion for Counts (IDC) and its limit can be expressed in a closed form.

The ideas which led to the proposed traffic model can be found in (Roberts et al. 1996) p. 338, but it seems rather difficult to mathematically analyze the pseudo self-similar traffic models based on Markov chains presented there.

The paper is organized as follows. Section 2 defines the traffic model which is the subject of this paper. In Section 3 the important properties of this process, characterizing long range dependence, are given. Section 4 derives an explicit expression for the IDC of this process and its limit. In Section 5 the behaviour of a queue whose 
input consists of this process is investigated. Section 6 illustrates the properties of the process by means of numerical examples. Conclusions are drawn in the last Section and also an outlook to future research is given.

\section{THE TRAFFIC MODEL}

\subsection{Definitions}

The traffic model that is envisaged is defined in the framework of Markovian Arrival Processes. For completeness reasons we recall the definition of a Discrete-Time Batch Markovian Arrival Process (D-BMAP), the discrete-time version of the BMAP defined in (Neuts 1989) and (Lucantoni 1991). (For more details, we refer the reader to (Blondia 1993)).

Consider a discrete-time Markov chain with transition matrix $\boldsymbol{D}$. Suppose that at time $k$ this chain is in some state $i, 1 \leq i \leq m$. At the next time instant $k+1$, there occurs a transition to another or possible the same state and a batch arrival may or may not occur. With probability $\left(d_{0}\right)_{i, j}, 1 \leq i \leq m$, there is a transition to state $j$ without an arrival, and with probability $\left(d_{n}\right)_{i, j}, 1 \leq i \leq m, n \geq 1$, there is a transition to state $j$ with a batch arrival of size $n$. We have that

$$
\sum_{n=0}^{\infty} \sum_{j=1}^{m}\left(d_{n}\right)_{i, j}=1
$$

Clearly the matrix $D_{0}$ with elements $\left(d_{0}\right)_{i, j}$ governs transitions that correspond to no arrivals, while the matrices $D_{n}$ with elements $\left(d_{n}\right)_{i, j}, n \geq 1$, govern transitions that correspond to arrivals of batches of size $n$.

The matrix $D=\sum_{n=0}^{\infty} D_{n}$ is the transition matrix of the underlying Markov chain. Let $\pi$ be stationary probability vector of this Markov process, i.e.

$$
\pi D=\pi, \quad \pi e=1,
$$

where $e$ is a column vector of 1 's.

The fundamental arrival rate $\lambda$ of this process is given by

$$
\lambda=\pi\left(\sum_{k=1}^{\infty} k D_{k}\right) e .
$$

A D-MAP is a special case of a D-BMAP, where arrivals have a batch of size 1 (for examples we refer to (Blondia 1993)).

Now we define the processes which are used to obtain the long range dependent process.

Consider a sequence $\left(X^{(i)}\right)_{i \in N}$ of independent on/off sources with the following characteristics. Let $1<b<a$. Assume that both the on and off period of the process $X^{(i)}$ are geometrically distributed with mean duration $\left(\frac{a}{b}\right)^{i}$, resp. $a^{i}$. While on, the source generates a cell in a slot with probability $p$, with $0<p<1$. Using matrix 
analytic notations, $X^{(i)}$ is a D-MAP with parameter matrices

$$
D_{0}^{(i)}=\left(\begin{array}{cc}
1-(1 / a)^{i} & (1 / a)^{i} \\
(1-p)(b / a)^{i} & (1-p)\left(1-(b / a)^{i}\right)
\end{array}\right)
$$

and

$$
\boldsymbol{D}_{1}^{(i)}=\left(\begin{array}{cc}
0 & 0 \\
p(b / a)^{i} & p\left(1-(b / a)^{i}\right)
\end{array}\right) .
$$

The matrix $D^{(i)}=D_{0}^{(i)}+D_{1}^{(i)}$ is the transition matrix of the underlying Markov chain of state transitions. The stationary distribution of $D^{(i)}$ is given by

$$
\pi^{(i)}=\left[\begin{array}{ll}
\frac{b^{i}}{\left(1+b^{i}\right)} & \frac{1}{\left(1+b^{i}\right)}
\end{array}\right] .
$$

The fundamental arrival rate $\lambda^{(i)}$ associated with $X^{(i)}$ is

$$
\lambda^{(i)}=\pi^{(i)} D_{1}^{(i)} e=p /\left(1+b^{i}\right) .
$$

From the definition of $X^{(i)}$ we see that for increasing $i$, both the on and off periods become longer. This property of the process $X^{(i)}$ will be responsible for the long range dependence of the envisaged process.

Let us now characterize the correlation structure of the process $X^{(i)}$. From (Blondia 1993), we know that

$$
\operatorname{Cov}\left(X_{1}^{(i)}, X_{1+k}^{(i)}\right)=\pi^{(i)} D_{1}^{(i)}\left(\left(D^{(i)}\right)^{k-1}-e \pi^{(i)}\right) D_{1}^{(i)} e
$$

Hence one can easily verify that

$$
\operatorname{Cov}\left(X_{1}^{(i)}, X_{1+k}^{(i)}\right)=\left(1-\left(\frac{1}{a}\right)^{i}-\left(\frac{b}{a}\right)^{i}\right)^{k} \frac{p^{2} b^{i}}{\left(1+b^{i}\right)^{2}} .
$$

In view of (Blondia 1993), p. 8, we know that a finite superposition $Y^{(M)}=\sum_{i=1}^{M} X^{(i)}$ of D-MAPs is a D-BMAP determined by the matrices

$$
\begin{aligned}
C_{0}^{(M)} & =D_{0}^{(M)} \otimes D_{0}^{(M-1)} \otimes \cdots \otimes D_{0}^{(1)}, \\
& \vdots \\
C_{i}^{(M)} & =\sum_{k_{M}+\ldots+k_{1}=i} \bigotimes_{j=M}^{1} D_{k_{j}}^{(j)}, \\
\vdots & \\
C_{M}^{(M)} & =D_{1}^{(M)} \otimes D_{1}^{(M-1)} \otimes \cdots \otimes D_{1}^{(1)} .
\end{aligned}
$$

The superposition $Y^{(\infty)}$ is not a D-BMAP any longer, but since the $X^{(i)}$ are independent, the expressions for the fundamental arrival rate $\lambda^{(\infty)}$ and the covariance 
structure are given by:

$$
\lambda^{(\infty)}=\sum_{i=1}^{\infty} \frac{p}{1+b^{i}}
$$

and

$$
\operatorname{Cov}\left(Y_{1}^{(\infty)}, Y_{1+k}^{(\infty)}\right)=\sum_{i=1}^{\infty}\left(1-\left(\frac{1}{a}\right)^{i}-\left(\frac{b}{a}\right)^{i}\right)^{k} \frac{p^{2} b^{i}}{\left(1+b^{i}\right)^{2}}
$$

\section{PROPERTIES OF THE PROCESS $Y^{(\infty)}$}

In this section the influence of the parameters $a$ and $b$ on the correlation structure of the arrival proces $Y^{(\infty)}$ is examined.

Property 1 The arrival process $Y^{(\infty)}$ is long range dependent if and only if $b^{2} \leqslant a$.

Proof. Following definition 13.4.1 (Roberts et al. 1996, page 326) we have long range dependence if and only if the series is

$$
\sum_{k=1}^{\infty} \operatorname{Cov}\left(Y_{1}^{(\infty)}, Y_{1+k}^{(\infty)}\right)
$$

diverges. Using (1) and changing the order of summation we deduce that the series (2) diverges if and only if

$$
\sum_{i=1}^{\infty} \frac{a^{i} b^{i}-b^{i}-b^{2 i}}{\left(1+b^{i}\right)^{3}}=\infty
$$

This series is similar to a geometric one and hence it diverges if and only if $b^{2} \leqslant a$.

Property 2 There exist $0<C_{1}<C_{2}<\infty$ such that

$$
C_{1} k^{-\beta}<\operatorname{Cov}\left(Y_{1}^{(\infty)}, Y_{1+k}^{(\infty)}\right)<C_{2} k^{-\beta}
$$

with

$$
\beta=\frac{\log b}{\log a-\log b} .
$$

Proof. See appendix.

Now we state the main result of this section, namely an explicit expression for the Hurst parameter of the process $Y^{(\infty)}$. 
Property 3 The Hurst parameter $H$ of the discrete-time arrival process $Y^{(\infty)}$ is given by

$$
H=\frac{1}{2}\left(2-\frac{\log b}{\log a-\log b}\right) .
$$

Proof. Based on (4), we see that $\operatorname{Cov}\left(Y_{1}^{(\infty)}, Y_{1+k}^{(\infty)}\right)$ decreases as $k^{-\beta}$, with $\beta=$ $\frac{\log b}{\log a-\log b}$. Hence, from (Roberts et al. 1996, page 327), we immediately conclude that $H=\frac{1}{2}\left(2-\frac{\log b}{\log a-\log b}\right)$.

Clearly, if $b^{2} \leqslant a$, then the Hurst parameter satisfies $\frac{1}{2} \leq H<1$, a criterion for long range dependence of the process $Y^{(\infty)}$.

In Section 5, the above properties are illustrated through numerical examples.

\section{THE INDEX OF DISPERSION FOR COUNTS OF THE TRAFFIC MODEL}

In this section we investigate the correlation structure of the process $Y^{(\infty)}$ by means of the Index of Dispersion for Counts (IDC).

Denote $N_{k}$ the number of arrivals in an interval of length $k$. The Index of Dispersion for Counts (IDC) at time $k$ is defined to be the variance of the number of arrivals in an interval of length $k$ divided by the the mean number of arrivals in this interval, i.e.

$$
I(k)=\frac{\operatorname{Var}\left(N_{k}\right)}{\mathrm{E}\left(N_{k}\right)} \text {. }
$$

It is well known that for a renewal process $I(k)=c_{1}^{2}$, for all $k \geq 1$, where $c_{1}^{2}$ is the squared coefficient of variation of the number of arrivals in a slot. In particular for a Bernoulli process, $I(k)=1$, for all $k \geq 1$.

Denote $I^{(i)}(k)$ the IDC of the process $X^{(i)}$ with $\lim _{k \rightarrow \infty} I^{(i)}(k)=J^{(i)}$ and $I^{(\infty)}(k)$ the IDC of the process $Y^{(\infty)}$, with $\lim _{k \rightarrow \infty} I^{(\infty)}(k)=J^{(\infty)}$.

From (Blondia \& Geerts 1997), we know that

$$
J^{(i)}=\frac{\pi^{(i)} D_{1}^{(i)} e-3\left[\pi^{(i)} D_{1}^{(i)} e\right]^{2}+2 \pi D_{1}^{(i)} Z^{(i)} D_{1}^{(i)} e}{\pi^{(i)} D_{1}^{(i)} e},
$$

with $Z^{(i)}$ the fundamental matrix of the Markov chain $D^{(i)}=D_{0}^{(i)}+D_{1}^{(i)}$, given by

$$
Z^{(i)}=\left[I-\left(D^{(i)}-e \pi^{(i)}\right)\right]^{-1} \text {. }
$$

From the expressions for $D_{0}^{(i)}$ and $D_{1}^{(i)}$ given in Section 2, it is easy to show that

$$
Z^{(i)}=\frac{1}{\left(1+b^{i}\right)^{2}}\left(\begin{array}{cc}
a^{i}+b^{i}\left(1+b^{i}\right) & 1-a^{i}+b^{i} \\
b^{i}\left(1-a^{i}+b^{i}\right) & 1+b^{i}+a^{i} b^{i}
\end{array}\right) .
$$


Hence,

$$
\pi^{(i)} D_{1}^{(i)} Z^{(i)} D_{1}^{(i)} e=\frac{p^{2}}{\left(1+b^{i}\right)^{3}}\left[1+b^{i}\left(a^{i}-b^{i}\right)\right] .
$$

Using this expression in (7), we obtain that

$$
J^{(i)}=1-3 \frac{p}{1+b^{i}}+2 \frac{p}{\left(1+b^{i}\right)^{2}}\left[1+b^{i}\left(a^{i}-b^{i}\right)\right] .
$$

Now we compute $J^{(\infty)}$, i.e. the limit of the IDC of the process $Y^{(\infty)}$. Since $Y^{(\infty)}=\sum_{i=1}^{\infty} X^{(i)}$, we have that $I^{(\infty)}(k)=\sum_{i=1}^{\infty} I^{(i)}(k)$. Hence,

$$
I^{(\infty)}(k)=\frac{\sum_{i=1}^{\infty} \operatorname{cov}\left(X_{1}^{(i)}, X_{1}^{(i)}\right)+\sum_{i=1}^{\infty} 2 \sum_{j=1}^{k-1} \frac{k-j}{k} \operatorname{cov}\left(X_{1}^{(i)}, X_{1+j}^{(i)}\right)}{\sum_{i=1}^{\infty} \mathrm{E}\left[X_{1}^{(i)}\right]}
$$

Taking the limit for $k \longrightarrow \infty$, we obtain

$$
J^{(\infty)}=\frac{\lambda^{(\infty)}-3 \sum_{i=1}^{\infty}\left(\lambda^{(i)}\right)^{2}+2 p^{2} \sum_{i=1}^{\infty} \frac{1+b^{i}\left(a^{i}-b^{i}\right)}{\left(1+b^{i}\right)^{3}}}{\lambda^{(\infty)}} .
$$

From equation (9) it follows that the limit of the IDC of the process $Y^{(\infty)}$ is infinite if $b^{2} \leqslant a$, which is exactly the condition under which the process has the long range dependence property (see Property 1 in Section 3). This is in agreement with the criterion that a process is long range dependent if its IDC is diverging.

\section{QUEUEING BEHAVIOUR}

We consider a queue of the G/D/1-type which has the arrival proces $Y^{(\infty)}$ as input. It turns out that the mean queue length is $\infty$. This result is obtained by studying the sequence of queues with arrival processes $\sum_{i=1}^{M} X^{(i)}$. These queues are of the D-BMAP/D/1-type. Consider the D-BMAP/D/1-queue with arrival proces $Y^{(M)}=$ $\sum_{i=1}^{M} X^{(i)}$. From now on we will drop the index $M$ to keep the notation simple.

The stationary queue distribution $\boldsymbol{x}$ of the D-BMAP/D/1 queue satisfies the following steady state equations

$$
\boldsymbol{x}=\left(\boldsymbol{x}_{0}, \boldsymbol{x}_{1}, \ldots\right)=\left(\boldsymbol{x}_{0}, \boldsymbol{x}_{1}, \ldots\right)\left(\begin{array}{cccc}
D_{0} & D_{1} & D_{2} & \ldots \\
D_{0} & D_{1} & D_{2} & \ldots \\
0 & D_{0} & D_{1} & \ldots \\
0 & 0 & D_{0} & \ldots \\
\vdots & \vdots & \vdots & \ddots
\end{array}\right)
$$

together with

$$
\boldsymbol{x} e=1 .
$$

This leads to

$$
X(z)(z I-D(z))=(z-1) x_{0} D(z)
$$


with generating functions $\boldsymbol{X}(z)=\sum_{n=0}^{\infty} \boldsymbol{x}_{n} z^{n}$ and $\boldsymbol{D}(z)=\sum_{n=0}^{\infty} \boldsymbol{D}_{n} z^{n}$. It is important to notice that $D(z)=D^{(M)}(z) \cdots D^{(1)}(z)$. The mean queue length is given by the expression $\boldsymbol{X}^{\prime} \boldsymbol{e}$ with $\boldsymbol{X}^{\prime}=\left.\frac{d}{d z} \boldsymbol{X}(z)\right|_{z=1}$. The computations made in (Lucantoni 1991) result in the following expression for the mean queue length

$$
X^{\prime} e=\frac{\left(\pi D^{\prime \prime} e+2 x_{0} D^{\prime} e-2 \rho+\left(2 x_{0} D+2 \pi D^{\prime}\right)(I-D+e \pi)^{-1} D^{\prime} e\right)}{2(1-\rho)},
$$

with $D=D(1), D^{\prime}=\left.\frac{d}{d z} D(z)\right|_{z=1}, D^{\prime \prime}=\left.\frac{d^{2}}{d z^{2}} D(z)\right|_{z=1}$ and $\pi=\pi^{(M)} \otimes \cdots \otimes \pi^{(1)}$ and $\rho=\sum_{i=1}^{M} \lambda^{(i)}$.

We show that the right hand side of (13) is diverging for $M \longrightarrow \infty$.

Since $\pi D^{\prime \prime} e>0$ and $2 x_{0} D^{\prime} e>0$, and as the load $\rho$ is bounded by some number, independent from $M$, it is sufficient to investigate the behaviour of the factor

$$
2 x_{0} D(I-D+e \pi)^{-1} D^{\prime} e+2 \pi D^{\prime}(I-D+e \pi)^{-1} D^{\prime} e
$$

for $M \longrightarrow \infty$. First we notice that

$$
(I-(D-e \pi))^{-1}=I+\sum_{k=1}^{\infty}\left(D^{k}-e \pi\right) .
$$

Furthermore, since

$$
\operatorname{Cov}\left(X_{1}, X_{1+k}\right)=\pi D^{\prime}\left(D^{k-1}-e \pi\right) D^{\prime} e
$$

we have

$$
\pi D^{\prime}\left(\sum_{k=1}^{\infty}\left(D^{k}-e \pi\right)\right) D^{\prime} e=\sum_{k=2}^{\infty} \sum_{i=1}^{M}\left(1-\left(\frac{1}{a}\right)^{i}-\left(\frac{b}{a}\right)^{i}\right)^{k} \frac{b^{i}}{\left(1+b^{i}\right)^{2}}
$$

The behaviour of $x_{0} D\left(\sum_{k=1}^{\infty}\left(D^{k}-e \pi\right)\right) D^{\prime} e$ is a bit more elaborated. From (Lucantoni 1991), we know that $x_{0}=(1-\rho) g$, with $g$ the steady state vector of the matrix $G$, describing the first passage times from one level to another. Hence,

$$
x_{0}=(1-\rho) u^{(M)} \otimes u^{(M-1)} \otimes \cdots \otimes u^{(1)}
$$

with $u^{(i)}$ the first row of the matrix $D^{(i)}$. Using the elementary properties of the Kronecker product $\otimes$, one obtains

$$
x_{0} D\left(\sum_{k=1}^{\infty}\left(D^{k}-e \pi\right)\right) D^{\prime} e=-(1-\rho) \sum_{k=1}^{\infty} \sum_{i=1}^{M}\left(1-\left(\frac{1}{a}\right)^{i}-\left(\frac{b}{a}\right)^{i}\right)^{k} \frac{1}{1+b^{i}}
$$

Hence, the mean queue lenght is diverging iff

$$
\lim _{M \rightarrow \infty} \sum_{i=1}^{M} \sum_{k=0}^{\infty}\left(1-\left(\frac{1}{a}\right)^{i}-\left(\frac{b}{a}\right)^{i}\right)^{k}\left(\frac{b^{i}}{\left(1+b^{i}\right)^{2}}-(1-\rho) \frac{1}{1+b^{i}}\right)=\infty .
$$

One can check this is the case iff $b^{2} \leqslant a$, in other words, iff the arrival process 


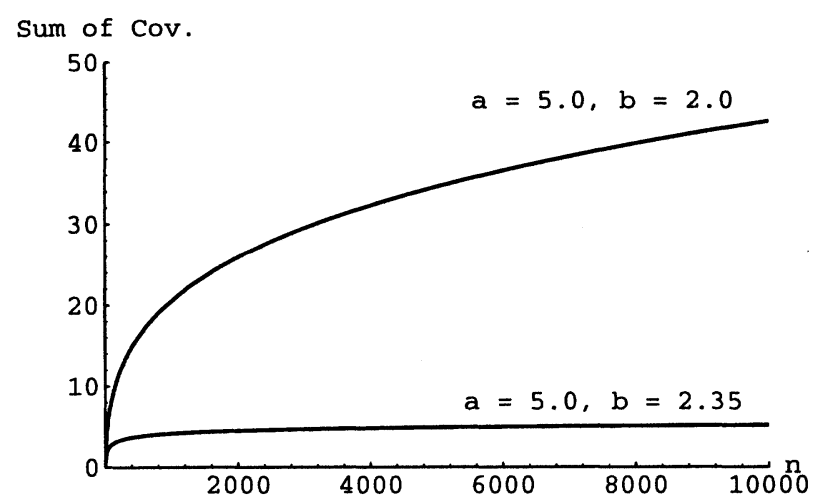

Figure 1 Influence of $a$ and $b$ on sum of covariances.

$Y^{(\infty)}$ is long range dependent. This result is in accordance with the one obtained in (Likhanov et al. 1995).

\section{NUMERICAL EXAMPLES}

\subsection{Example 1}

In this example we illustrate Property 1 of Section 3. Consider two superpositions of on/off sources, the first with parameters $a_{1}=5$ and $b_{1}=2$ and the second with parameters $a_{2}=5$ and $b_{2}=2.35$. Application of Property 1 immediately shows that contrary to the second superposition, the first superposition is long range dependent (as $b_{1}^{2} \leqslant a_{1}$ ). This is illustrated in Figure 1 , where the sum of covanriances of the first superposition clearly does not converge, while the second superposition does.

\subsection{Example 2}

In this example we consider the processes $Y^{(M)}=\sum_{i=1}^{M} X^{(i)}$. We illustrate the influence of the value $M$ on the behaviour of the sum of covariances $\sum_{k=1}^{n} \operatorname{Cov}\left(Y_{1}^{(M)}, Y_{k+1}^{(M)}\right)$ of increasing $n$. 


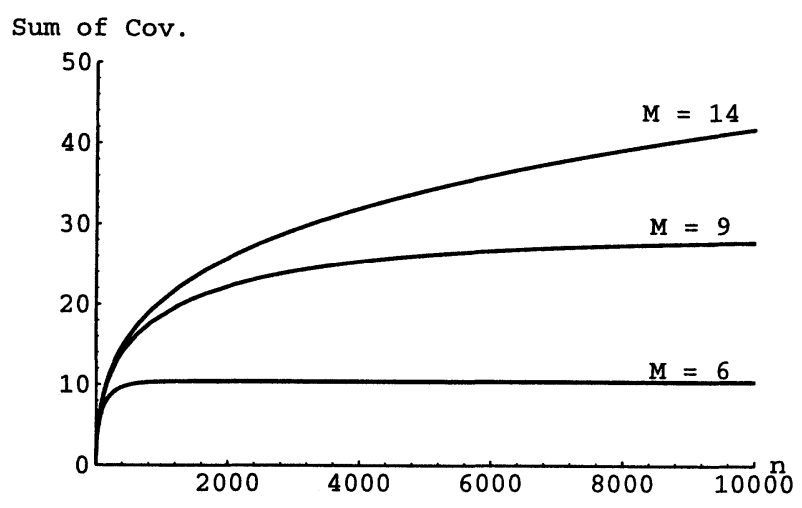

Figure 2 Influence of $M$ on sum of covariances.

Let $M=6,9,14$. In Figure 2, we see that for higher values of $M$, the convergence of $\sum_{k=1}^{n} \operatorname{Cov}\left(Y_{1}^{(M)}, Y_{k+1}^{(M)}\right)$ is slower than for smaller values. This result is in accordance with Property 2 of Section 3, which states that the sum is divergent for $M=\infty$.

\section{CONCLUSIONS AND FUTURE RESEARCH}

In this paper we have introduced a discrete-time traffic model resulting from the superposition of a sequence of on/off sources with increasing on and off period duration. Under a simple condition, the traffic model exhibits a long range dependence character. Moreover the Hurst parameter can be computed explicitly. Queueing problems in which this process is involved can be easily handled by considering a matrix-analytic approach. The correlation structure of the process is investigated by means of the IDC and its limit. Here again closed form formulas are obtained.

The proposed process will be used in future research to investigate the influence of long range dependent traffic on delays and loss probabilities in queueing systems when merging with Markovian traffic. Furthermore we will investigate how to choose the parameters when matching this process with data obtained from measurements. 


\section{ACKNOWLEDGEMENT}

The first author is "aspirant" with the Science Foundation of Flanders (FWO), hence the authors would like to thank this institute for the support of this research. This work was also supported by the European Research Programme ACTS, under project AC094 EXPERT.

\section{REFERENCES}

Andersen, A. T. (1995), Modelling of packet traffic with matrix analytical methods, $\mathrm{PhD}$ thesis, Technical University of Denmark.

Andersen, A. T., Jensen, A. \& Nielsen, B. F. (1995), Modelling and performance study of packet traffic with self-similar characteristics over several timescales with Markovian Arrival Processes (MAP), in I. Norros \& J. Virtamo, eds, 'Nordic Teletraffic Seminar', Espoo, Finland.

Andersen, A. T. \& Nielsen, B. F. (n.d.), An application of superpositions of two state Markovian sources to the modelling of self-similar behaviour. Preprint 97.

Anick, D., Mitra, D. \& Sondhi, M. M. (1982), 'Stochastic theory of a datahandling system with multiple sources', The Bell System technical Journal 61(8), 1871-1894.

Beran, J., Sherman, R., Taqqu, M. \& Willinger, W. (1995), 'Long-range depedence in variable-bit-rate video traffic', IEEE Transactions on Communications 43(2/3/4).

Blondia, C. (1993), 'A discrete-time batch markovian arrival process as B-ISDN traffic model', Belgian Journal of Operations Research, Statistics and Computer Science 32.

Blondia, C. \& Geerts, F. (1997), The correlation structure of the output of an ATM multiplexer, in 'Proceedings of the fifth IFIP Workshop on Performance Modelling and Evaluation of ATM Networks', IFIP.

Fowler, H. J. \& Leland, W. E. (1991), 'Local area network traffic characteristics, with implications for broadband network congestion management', IEEE Journal on Selected Areas in Communications 9(7), 1139-1149.

Leland, W. E., Taqqu, M. S., Willinger, W. \& Wilson, D. V. (1994), 'On the selfsimilar nature of ethernet traffic (extended version)', IEEE/ACM Transactions on networking 2, 1-15.

Likhanov, N., Tsybakov, B. \& Georganas, N. D. (1995), Analysis of an ATM buffer with self-similar ("fractal") input traffic, in 'Proceedings of INFOCOM'95', IEEE.

Lucantoni, D. M. (1991), 'New results on the single server queue with a batch Markovian arrival process', Stochastic Models .

Neuts, M. F. (1989), Structured stochastic matrices of the M/G/1-type and their applications, Marcel Dekker. 
Robert, S. \& Le Boudec, J. (1995), A Markov modulated process for self-similar traffic, in 'Internationales Begegnungs und Forschungszentrum fuer Informatik'. Roberts, J., Mocci, U. \& Virtamo, J. (1996), Broadband network teletraffic, Springer. 


\section{APPENDIX}

An outline of the proof of property 2 is given. We restrict ourselves to the case $b^{2}<a$. The case $b^{2}=a$ can be proved in a similiar way. To simplify things we first study the behaviour of the function

$$
g(k)=\sum_{i=1}^{\infty}\left(1-\left(\frac{b}{a}\right)^{i}\right)^{k}\left(\frac{1}{b}\right)^{i}
$$

Because of

$$
\begin{aligned}
\int_{2}^{\infty}\left(1-(b / a)^{x-1}\right)^{k} 1 / b^{x} d x<\sum_{i=2}^{\infty}\left(1-(b / a)^{i}\right)^{k} 1 / b^{i} \\
<\sum_{i=1}^{\infty}\left(1-(b / a)^{i}\right)^{k} 1 / b^{i}<\int_{1}^{\infty}\left(1-(b / a)^{x-1}\right)^{k} 1 / b^{x-1} d x
\end{aligned}
$$

it suffices to examine the integral $\int_{1}^{\infty}\left(1-(b / a)^{x}\right)^{k} 1 / b^{x} d x$ in function of $k$. We rewrite it as the sum

$$
\int_{1}^{\frac{\log k}{\log a-\log b}}\left(1-(b / a)^{x}\right)^{k} 1 / b^{x} d x+\int_{\frac{\log k}{\log a-\log b}}^{\infty}\left(1-(b / a)^{x}\right)^{k} 1 / b^{x} d x .
$$

From now on we denote $\log k /(\log a-\log b)$ by $\gamma_{k}$. Since $\left(1-(b / a)^{x}\right)^{k}<e^{-k(b / a)^{x}}<$ $1 / k(b / a)^{-x}$ we obtain a bound for the first integral in 21 as follows:

$$
\begin{aligned}
\int_{1}^{\gamma_{k}}\left(1-(b / a)^{x}\right)^{k} 1 / b^{x} d x & <\int_{1}^{\gamma_{k}} \frac{1}{k}\left(a / b^{2}\right)^{x} d x \\
& <\frac{1}{\log a-2 \log b} k^{-\beta}
\end{aligned}
$$

with $\beta=\log b /(\log a-\log b)$. For the second integral of 21 we use the inequality $\left(1-(b / a)^{x}\right)^{k}<1-k(b / a)^{x}+k^{2}(b / a)^{2 x} / 2$. Hence

$$
\begin{aligned}
\int_{\gamma_{k}}^{\infty}\left(1-(b / a)^{x}\right)^{k} 1 / b^{x} d x< & \int_{\gamma_{k}}^{\infty}\left(1-k(b / a)^{x}\right) 1 / b^{x} d x \\
& +\int_{\gamma_{k}}^{\infty} k^{2} / 2(b / a)^{2 x} 1 / b^{x} d x \\
= & \left(\frac{1}{\log b}-\frac{1}{\log a}\right) k^{-\beta}+\frac{k^{-\beta}}{2 \log a-\log b}
\end{aligned}
$$

Finally, by using the inequality $1-k(b / a)^{x}<\left(1-(b / a)^{x}\right)^{k}$, we obtain

$$
\begin{aligned}
& \frac{1}{b}\left(\frac{1}{\log b}-\frac{1}{\log a}\right) k^{-\beta}<g(k) \\
& \quad<\left(\frac{1}{\log a-2 \log b}+\frac{1}{\log b}-\frac{1}{\log a}+\frac{1}{2 \log a-\log b}\right) b k^{-\beta} .
\end{aligned}
$$


If we drop the $p^{2}$ in 1 , it is easy to see that

$$
\operatorname{Cov}\left(Y_{1}^{(\infty)}, Y_{k+1}^{(\infty)}\right)<g(k)
$$

Some algebraic computations lead to the following inequality

$$
g(k)-\operatorname{Cov}\left(Y_{1}^{(\infty)}, Y_{k+1}^{(\infty)}\right)<\sum_{i=1}^{\infty}\left(1-(b / a)^{i}\right)^{k} \frac{3}{b^{2 i}}+\sum_{i=1}^{\infty} k\left(1-(b / a)^{i}\right)^{k} \frac{1}{b^{i}} \frac{1}{a^{i}}
$$

By using similar techniques as before it is possible to show

$$
g(k)-\operatorname{Cov}\left(Y_{1}^{(\infty)}, Y_{k+1}^{(\infty)}\right)<C k^{-(\beta+\delta)}
$$

for $\delta>0$ such that $b^{2+\delta}<a$ and some $C>0$. 